\title{
MS30-02 | Electron Crystallography for Determining the Handedness of ChiRAL
}

\section{CRYSTALS}

Oleynikov, Peter (ShanghaiTech University, Shanghai, CHN); Ma, Yanhang (ShanghaiTech University, Shanghai, CHN); Terasaki, Osamu (ShanghaiTech University, Shanghai, CHN)

The atomic level chirality study of a sub- $\mu$ m-sized crystal is a difficult task due to the lack of efficient and reliable characterization methods. Here, we propose two novel and practical electron crystallography approaches of characterization for determining the handedness of chiral zeolite nanocrystals [1]. The handedness is evaluated either by (i) the comparison of two aligned high-resolution transmission electron microscope images taken along 2 different zone axes of the same nanocrystal by tilting it around its screw axis, or (ii) the analysis of intensity asymmetry for a Bijvoet pair of reflections in a single precession electron-diffraction pattern. The image alignment is performed using gold nanoparticle markers; depending on the tilt direction the observable shift between the two projections leads to the correct space group assignment. The asymmetry in the diffraction spots intensities is analyzed by quantitative comparison with calculated dynamical electron diffraction patterns. Both approaches provide new elegant ways for the handedness determination of small, chiral crystals.

[1] Y.H. Ma, P. Oleynikov, O. Terasaki. Electron Crystallography for Determining the Handedness of Chiral Crystals. Nature Materials. 16(7) (2017) 755-759. 